\title{
Ill-conditioned linear equations and its algorithm
}

\author{
Dazhou Wang ${ }^{1, \mathrm{a}}$ Yueguang $\mathrm{Li}^{2, \mathrm{~b}}$ \\ ${ }^{1}$ Gansu Normal University for Nationalities, Hezuo, gansu, 747000, \\ China \\ ${ }^{2}$ Gansu Normal University for Nationalities, Hezuo, gansu, 747000, \\ China \\ 'Yueguangli7@163.com, ${ }^{b}$ Yueguangli7@sohu.com
}

\begin{abstract}
In this paper, according to the characteristics of ill-conditioned linear equations. An improved Wilkinson algorithm(IWA) for solving ill-conditioned linear equations is proposed. An amendment factor is introduced to reduce the condition number of the coefficient matrix of ill-conditioned linear equations. An automatic step size is adopted to estimate the local error and change the step size correspondingly. The numerical results demonstrate that this new iterative algorithm is superior to other methods such as the amended conjugate gradient. The new algorithm is more applicable for solving ill-conditioned linear equations. Keywords: Wilkinson algorithm; ill-conditioned linear equations; step size; amended conjugate gradient
\end{abstract}

\section{Introduction}

In practical science and engineering applications, many problems arise in solving linear equations using approaches such as spline interpolation, finite differences for solving partial differential equations, finite element method, and the boundary element method. Over the years, many researchers have carried out extensive and detailed research into this problem and obtained many valuable results ${ }^{[1-6]}$. Because of various error forms, such as model errors, measurement errors and computational rounding errors, there is a certain degree of variation in the coefficient matrix and non-homogeneous information is obtained. As different methods use different iterative mechanisms, the error in the solving process accumulates to different degrees and leads to different approximations of the solution for different methods. In particular, for ill-conditioned linear equations, many of the existing algorithms do not work well. Based on Wilkinson's iterative algorithm and the Wu and Fang improved algorithm, a new algorithm with automatic step size is proposed here. An amendment factor is 
introduced to change the condition number of linear equations. By solving general linear equations of good condition, as well as high-order ill-conditioned Hilbert linear equations, numerical experiments demonstrate that the new algorithm is practical and more effective.

\section{PROBLEM FOR EMULATION}

We consider the linear equations defined by

$A X=B$

Equation (1) can be also written as

$\sum_{j=1}^{n} a_{i j} x_{j}=b_{i} \quad i=1,2, \ldots, m$

Where matrix $\quad A=\left(a_{i j}\right)_{m * n} \in R^{m * n} \quad$,and $A \neq 0$, vector $B=\left(b_{1}, b_{2}, \ldots, b_{m}\right)^{T} .\|A\|_{v}(v=1,2, \ldots, \infty)$ is the v-norm of matrix A. Here

$\operatorname{cond}(A)_{V}=\|A\|_{V}\left\|A^{-1}\right\|_{V}$

We define $\operatorname{cond}(A)_{V}=\|A\|_{V}\left\|A^{-1}\right\|_{V} \operatorname{Cond}(\mathrm{A})$ vas thev-condition number of matrix A. Especially, when $v=2$, since

$\sigma_{\max }=\sqrt{\lambda_{\max }\left(A^{T} A\right)}$

$\sigma_{\min }=\sqrt{\lambda_{\min }\left(A^{T} A\right)}$

Hence $\operatorname{cond}(A)_{2}=\sigma_{\max } / \sigma_{\min }$

Especially, if $A=1$, $\operatorname{cond}(A)_{2}=1$; if $A$ is a singular matrix, $\operatorname{Cond}(A)_{2}=\infty$.

\section{Improved Wilkinson algorithm (IWA)}

In 1971, Wilkinson proposed the following iterative algorithm based on the numerical solution of ordinary differential equations. Assume that the initial vector is $\mathrm{x}_{0}=0$, then its iterative formula is:

$$
A_{Z n}=b-A x_{n} \quad n=0,1,2, \ldots
$$




$$
Z_{n}=A^{-1}\left(b-A x_{n}\right) \quad n=0,1,2, \ldots
$$

In fact, Wilkinson's iterative algorithm is a Euler method with fixed step $Z_{\mathrm{n}} \equiv 1$ for solving ordinary differential equations:

$$
\left\{\begin{array}{l}
\frac{d_{x}}{d_{t}}=A^{-1}\left(b-A x_{n}\right) \\
x(0)=x_{0}=0
\end{array}\right.
$$

However, the error for an explicit Euler method with a fixed step may still be great when solving some equations, and the iterative convergence can also be very slow. Cholesky decomposition can be used for each step of Wilkinson's iterative algorithm. For a large-scale matrix, this not only increases the amount of computation, but also decreases the precision. In particular, for high-order ill-conditioned linear equations, we usually cannot obtain a satisfactory answer, no matter how many steps are set. Wu and Fang ${ }^{[1]}$ proposed an improved algorithm with automatic step size using a Euler formula and a trapezoid formula for solving linear ordinary differential equations. The main process of their improved algorithm is as follows. First, for linear ordinary differential Eq. (9), the following formula can be obtained using an explicit Euler method:

$$
x_{n+1}=x_{n}+Z_{n} A^{-1}\left(b-A x_{n}\right) \quad n=0,1,2, \ldots
$$

And the following formula is obtained using the trapezoid formula:

$$
x_{n+1}=x_{n}+\frac{Z_{n}}{2} A^{-1}\left(2 b-A\left(x_{n}+x_{n=1}\right)\right) \quad n=0,1,2, \ldots
$$

Wu and Fang proved the convergence of the iteration. Using both Wilkinson's iterative algorithm and the $\mathrm{Wu}$ and Fang improved algorithm, $Z_{\mathrm{n}}$ is obtained by solving linear equations of the form $A Z_{n}=b-A x_{n}$ in each step of the iteration. However, this is very difficult for high-order ill-conditioned linear equations and the error is also great, and both iterative algorithms fail for high-order Hilbert linear equations. In general, an n-order Hilbert linear equation takes the form

$\sum_{j=1}^{n} a_{i j} x_{j}=b_{i} \quad i=1,2, \ldots, n$

Where

$$
a_{i j}=\frac{1}{i+j-1} \quad(i, j=1,2, \ldots, n)
$$

,and $b_{i}=\sum_{k=1}^{n} k a_{i k} \quad i=1,2, \ldots, n$.Obviously, the solution accuracy of Eq. (12) is 
$x=[1,2, \ldots, n]^{\mathrm{T}}$. If we let $n=100$ and assume the upper bound of the iterative number is 10000 , we can use the improved algorithm proposed by $\mathrm{Wu}$ and Fang combined with Cholesky decomposition ${ }^{[7]}$ or the amended conjugate gradient method $^{[8]}$ to solve Eq. (12). The results shown in Table 1 demonstrate that both algorithms are not suitable for solving high-order ill-conditioned Hilbert linear equations.

Table 1 Comparison of the solution accuracy for the Wu and Fang improved algorithm

\begin{tabular}{|c|c|c|}
\hline \multirow{2}{*}{$n$} & \multicolumn{2}{|c|}{ Accuracy of the Wu and Fang solution } \\
\cline { 2 - 3 } & $\begin{array}{c}\text { With Cholesky } \\
\text { decomposition }\end{array}$ & $\begin{array}{c}\text { With amended conjugate } \\
\text { gradient }\end{array}$ \\
\hline 1 & 0.999996484266 & 1.00240968371554 \\
\hline 2 & 2.000459468168 & 1.944307180417133 \\
\hline 3 & 2.985326227504 & 3.30494636184830 \\
\hline 4 & 4.195908810628 & 3.450279752535835 \\
\hline 5 & -3.01003688033 & 5.12481410190494 \\
\hline$\ldots$ & $\ldots$ & $\ldots$ \\
\hline 99 & 97.757269770480 & 98.13845741566571100 \\
\hline 100 & 93.246322198282 & 98.8892193560027 \\
\hline
\end{tabular}

\section{The analysis of simulation}

Experimental selection of the 3 equations, the equation groups is used in literature [9].

$$
\begin{aligned}
& \left\{\begin{array}{l}
f_{1}(x)=\left(x_{1}-5 x_{2}\right)^{2}+40 \sin ^{2}\left(10 x_{3}\right) \\
f_{2}(x)=\left(x_{2}-2 x_{3}\right)^{2}+40 \sin ^{2}\left(10 x_{1}\right) \quad-1 \leq x_{i} \leq 1 \\
f_{3}(x)=\left(3 x_{1}+x_{3}\right)^{2}+40 \sin ^{2}\left(10 x_{2}\right)
\end{array}\right. \\
& \left\{\begin{array}{l}
f_{1}(x)=\left(x_{1}-5 x_{2}\right)^{2} \\
f_{2}(x)=\left(x_{2}-2 x_{3}\right)^{2} \\
f_{3}(x)=\left(3 x_{1}+x_{3}\right)^{2}
\end{array} \quad-1 \leq x_{i} \leq 1\right. \\
& \left\{\begin{array}{l}
f_{1}(x)=x_{1}+x_{2}+x_{3}-0.5 \\
f_{2}(x)=x_{1} x_{2}+x_{2} x_{3}+x_{3} x_{1}+7.5 \\
f_{3}(x)=x_{1}^{2}+x_{2}^{2}+x_{3}^{2}-15.25
\end{array}\right.
\end{aligned}
$$


In order to test the algorithm of the paper efficiency, in this paper, the algorithm of the paper compares to the algorithm of literature [10]. It can be seen from Figure 1, with the quantum-behaved particle swarm optimization (QBPSO) being premature and waggle-ness in the later stage of evolution, it generates large errors of numerical solution for solving ill-conditioned linear equation group. But the result of numerical solution by improved Wilkinson algorithm (IWA) is improved in comparison with quantum-behaved particle swarm optimization (QBPSO). Tests on the nuclear magnetic resonance oil well logging system of equations prove that the improved Wilkinson algorithm (IWA) proposed in this paper is an effective algorithm.

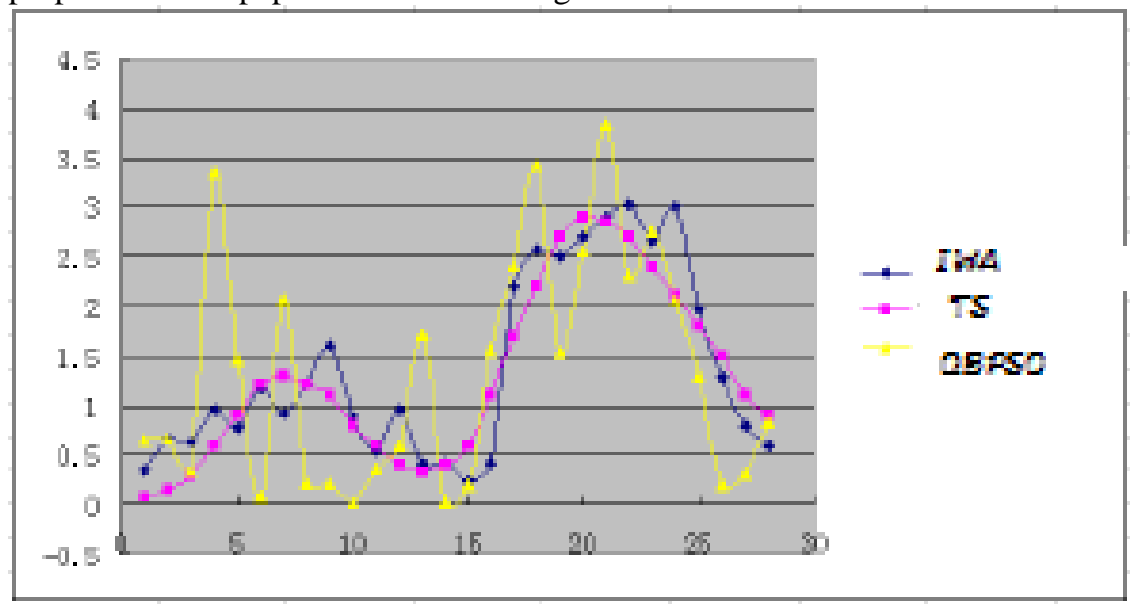

Figure 1 the algorithm of the paper compares to the algorithm of literature

\section{Conclusions}

This paper proposes an improved Wilkinson algorithm (IWA). The improved Wilkinson algorithm (IWA) is applied to solve ill-conditioned linear equations, the performance have been improved obviously. Experiments show that: the algorithm is feasible and effective for solving nonlinear equations.

\section{Acknowledgment}

This work is supported by Gansu Provincial College graduate tutor of scientific research project (1112-09) and Dean Fund of Gansu Normal College for Nationalities (09-07).

\section{References}

[1] X. Wu, Y. Fang, Wilkinson's iterative refinement of solution with automatic step-size control for linear system of equations. Appl. Math. Computer. 193:506-513 (2007). 
[2] X. Lai, G. Tan, Y. Zhou, Using genetic algorithms of large-scale linear ill-conditioned equations. J. Shanghai Normal Coll. 26(6):85-88(2006).

[3] W. Shi, 0.618 applied in solving linear equations. Coll. Math.19(3):77-82 (2003).

[4] X. Zheng, D. Zhang, Determination of ill-conditioned linear equations method. Mathematics in practice and theory. 36(9):166-170 (2006).

[5] S. Hu, New method for solving ill-conditioned linear equations: error transfer. J. South China Agric. Univ. 22(4):92-95 (2001).

[6] W. Zhang, Multi-step decline method with memory for solving ill-conditioned linear equations. Journal of Shanghai maritime university. 25(3):16-20 (2004).

[7] R.S. Martin, G. Peters, J.H. Wilkinson, Iterative refinement of the solution of a positive definite system of equations, in Linear Algebra, Handbook for Automatic Computation, Vol. II, (F.L. Baner, Ed.),Spring-Verlag, Berlin, 1971.

[8] W. Shi et al., New method for solving linear equations. Chin. J.Comput. Mech. 20(6):65-69 (2003).

[9] Guo Haiyan, Jin Xin, Hu Xiaobing. Research on the solving of nonlinear equation group based on swarm particle optimization[J]. Computer Engineering and Applications, 2006(15):72-74.

[10] Zhao Ji, Xu Wen-bo, Sun Ju. Multi-peaks function optimization using quantum-behaved particle swarm optimization[J]. Computer Applications, 2006(26):55-59. 